\title{
Factors That Affect Adolescent Drug Users' Suicide Attempts
}

\author{
Subin Park ${ }^{1}$ and Hokwang Song ${ }^{2}$ \\ ${ }^{1}$ Department of Research and Planning, National Center for Mental Health, Seoul, Republic of Korea \\ ${ }^{2}$ Department of Child and Adolescent Psychiatry, National Center for Mental Health, Seoul, Republic of Korea
}

\begin{abstract}
Drug abuse has been widely linked to suicide risk. We examined the factors that affect adolescent drug users' suicide attempts in South Korea. This study analyzed the data of 311 adolescents who had used drugs such as inhalants, psychotropic drugs, and marijuana (195 males and 116 females). Among 311 subjects, 109 (35.0\%) had attempted suicide during the last 12 months. After adjusting for other variables, depressive mood $(\mathrm{OR}=19.79)$ and poly-drug use $(\mathrm{OR}=2.79)$, and low/middle levels of academic achievement compared with a high level $(\mathrm{OR}=3.72$ and 4.38) were independently associated with increased odds of a suicide attempt, while better perceived health $(\mathrm{OR}=0.32)$ was independently associated with reduced odds of a suicide attempt. For adolescent drug users, preventive work should be directed toward the active treatment of drug use, depression, and physical health and reinforcing proper coping strategies for academic and other stress.

Psychiatry Investig 2016;13(3):360-363
\end{abstract}

Key Words Drug use, Suicide, Risk factor, Adolescents.

\section{INTRODUCTION}

Adolescent suicide, the third leading cause of death among youths 15 to 19 years of age, is a serious public health problem. ${ }^{1}$ South Korea has the highest suicide rate as reported by the Organization for Economic Cooperation and Development (OECD), and suicide rates have increased steadily in recent years among Korean adolescents. ${ }^{2}$ This is contrary to a decreasing trend of suicide mortality among adolescents in other OECD countries. ${ }^{3}$ Many studies have shown substance use to be one of the most prominent risk factors associated with adolescent suicidal behavior., ${ }^{4,5}$

Risk and protective factors play a critical role in suicide prevention. ${ }^{6}$ For clinicians, identifying risk and protective factors provides critical information to assess and manage suicide risk in individuals. ${ }^{7}$ Identifying specific risk factors in high-risk groups, such as drug abusers, is particularly important because of the need for close monitoring and targeted interventions to prevent suicide.

Received: July 9, 2015 Revised: September 21, 2015

Accepted: October 2, 2015 Available online: March 23, 2016

$\triangle$ Correspondence: Subin Park, MD, PhD

Department of Research and Planning, National Center for Mental Health, 127 Yongmasan-ro, Gwangjin-gu, Seoul 04933, Republic of Korea

Tel: +82-2-2204-0108, Fax: +82-2-2204-0280, E-mail: subin-21@hanmail.net

(a) This is an Open Access article distributed under the terms of the Creative Commons Attribution Non-Commercial License (http://creativecommons.org/licenses/bync/3.0) which permits unrestricted non-commercial use, distribution, and reproduction in any medium, provided the original work is properly cited.
South Korea has been viewed as a relatively drug-free country compared to other countries worldwide. This is largely the result of the Korean government's policy of severe punishment for drug-related crimes, including simple drug abuse. However, since the 1990s, drug abuse has expanded in Korean society. Students studying abroad have played a key role in expanding the variety of available drugs, including LSD, Ecstasy, and other new drugs, such as potent analgesics. ${ }^{8}$

Considering the high suicide rates and rapid increase in drug use in the Korean adolescent population, it is important to understand the risk factors for suicide attempts in this highrisk group. Therefore, we examined the factors that affect adolescent drug users' suicide attempts in South Korea.

\section{METHODS}

\section{Subjects}

Out study is based on data from the 2014 Korean Youth Risk Behavior Web-Based Survey (KYRBS), collected from 72,060 adolescents aged $12-18$ years. The KYRBS has been conducted annually since 2005 by the Korea Centers for Disease Control and Prevention and the Ministry of Education, Science and Technology, in order to monitor the prevalence of the health behaviors among the adolescents. The data was collected with a web-based survey conducted on a nationally representative sample of middle and high school students with a stratified, clustered, multistage probability sampling design. 
Additional details on the sampling methodology and survey procedure are available elsewhere. ${ }^{9}$ The KYRBS was reviewed and approved by the institutional review board of the Korea Centers for Disease Control and Prevention (2014-06EXP02-P-A).

In the KYRBS, lifetime drug use was assessed with the following question: "Have you ever used drugs that are often used nonmedically (e.g., inhalants, glue, stimulants, cannabis, amphetamines, marijuana, codeine, neuroleptics) for mood elevation, hallucinatory experiences, or excessive dieting?" Participants who responded "Yes" to this question were asked to choose the drugs they had ever used from a list of 33 commonly used illegal drugs. Among the 72,060 adolescents participating in the KYRBS, 311 (0.43\%, 95\% CI 0.40-0.46) reported using any type of drug. This study analyzed the data of those 311 adolescents (195 males and 116 females; mean age $=15.95 \pm 1.41$ years).

\section{Measurements}

Suicide attempts were assessed with the question: "Have you attempted suicide during the past 12 months?"

Socio-demographic variables included sex, age, place of residence, school type, and residential type (living with family, with relatives, with friends/alone/in a dormitory, or in a facility). Perceived family economic status and perceived academic achievement were assessed by a 5 -point Likert scale. On the basis of the responses, the participants were classified into the following three groups: 1) low (1), 2) middle (2-4), and 3) high (5). Violence victimization was assessed with the question: "Have you been a victim of physical violence, threats of violence, or social exclusion during the past 12 months?"

The level of perceived health was measured with the following question: "How healthy do you usually feel?" The response options were very unhealthy (1), unhealthy (2), average (3), healthy (4), and very healthy (5). On the basis of the responses, participants were classified into the following two groups: 1) Saverage perceived health (1-3) and 2) >average perceived health $(4-5)$. The level of sleep satisfaction was measured with the following question: "In the last week, how satisfactory was your sleep in terms of relieving your fatigue?" The response options were very unsatisfactory (1), unsatisfactory (2), average (3), satisfactory (4), and very satisfactory (5). On the basis of the responses, participants were classified into the following two groups: 1) Saverage satisfaction with sleep (1-3) and 2) >average satisfaction with sleep (4-5). Depressive symptoms were measured with the following question: "During the past 12 months, did you ever feel so sad or hopeless almost every day for two weeks or more that you stopped doing some usual activities?" Problematic alcohol use during the last 12 months was assessed with the CRAFET, a 6-item instrument that is used to screen for alcohol use in the adolescent population. Two or more positive responses indicate the potential for a significant alcohol problem.

\section{Statistical analysis}

A univariate analysis was used to examine the association between suicide attempts and each of the potential protective and risk factors. Multiple logistic regression analyses then examined the association between each of the independent variables and the suicide attempt, controlling for the twelve potential confounders. For example, the multivariate odds ratio between suicide attempts and poly-drug use was calculated utilizing a regression model of poly-drug use predicting suicide attempts in the past year, controlling for gender, age, area of residence, school type, residential type, socio-economic status, academic achievement level, violence victimization, perceived health, perceived sleep satisfaction, depressive mood, and problematic alcohol use. All statistical analyses were performed using SPSS (version 21.0; SPSS Inc., Chicago, IL, USA), with statistical significance defined as an alpha level $<0.05$.

\section{RESULTS}

Among 311 subjects, 109 (35.0\%) had attempted suicide during the last 12 months. In the univariate analyses, low academic achievement $(\mathrm{OR}=2.38,95 \% \mathrm{CI}=1.32-4.30)$, violence victimization $(\mathrm{OR}=2.20,95 \% \mathrm{CI}=1.37-3.54)$, depressive $\operatorname{mood}(\mathrm{OR}=10.44,95 \% \mathrm{CI}=5.28-20.65)$, problematic alcohol use $(\mathrm{OR}=1.65,95 \% \mathrm{CI}=1.03-2.63)$, and poly-drug use $(\mathrm{OR}=$ $2.13,95 \% \mathrm{CI}=1.22-3.72$ ) were associated with a significantly increased likelihood of suicide attempt in adolescent drug users. Better perceived health was associated with a significantly decreased likelihood of a suicide attempt (OR=0.44, 95\% $\mathrm{CI}=0.27-0.71)$. In the multivariate analysis, depressive mood $(\mathrm{OR}=19.79)$ and poly-drug use $(\mathrm{OR}=2.79)$, and low/ middle levels of academic achievement compared with a high level $(\mathrm{OR}=3.72$ and 4.38) were independently associated with increased odds of a suicide attempt, while better perceived health $(\mathrm{OR}=0.32)$ was independently associated with reduced odds of a suicide attempt, after adjusting for other variables (Table 1).

\section{DISCUSSION}

The one-year prevalence of suicide attempts among adolescent drug users was 35\%, a level higher than that seen in other studies of drug users ranging from $12 \%$ to $15 \% .{ }^{10-12} \mathrm{Be}$ cause drug use is relatively very rare in Korean adolescents compared to Western countries, Korean drug users are more 
likely to have more severe mental health problems or be in a worse situation than drug users in Western countries. This may have affected the higher rate of past-year suicide attempts.

We found that depressive symptoms and poly-drug use were independent risk factors, whereas high academic achievement and better perceived health were independent protective factors for suicide attempts among adolescent drug users in South Korea. Violence victimization and problematic alcohol use were also associated with suicide attempts, but after adjusting for other variables, these associations did not remain significant. Because drug use itself is an important risk factor for suicide, drug-users with these additional risk factors may rep- resent an extremely high risk group and require close monitoring and proactive intervention.

Consistent with our results, Darke et al. ${ }^{13}$ found that the past 12-month suicide attempts among heroin users were independently associated with a current diagnosis of depression (OR 1.67) and more extensive polydrug use (OR 1.39), while better subjective health was associated with a reduced odds of suicide attempt (OR 0.66). It was reported that the extent of polydrug use was an independent correlate of suicidal behavior rather than the individual drugs comprising such use. ${ }^{14}$ According to the review by Pompili et al. ${ }^{15}$ adolescents with substance abuse disorder who attempt or complete suicide

Table 1. Odds ratios of predictors for suicide attempts in the past year among adolescent drug users

\begin{tabular}{|c|c|c|c|c|c|c|c|}
\hline & $\begin{array}{l}\text { Drug users } \\
(\mathrm{N}=311)\end{array}$ & $\begin{array}{c}\text { Suicide } \\
\text { attempters } \\
(\mathrm{N}=109)\end{array}$ & $\begin{array}{c}\text { Non- } \\
\text { attempters } \\
(\mathrm{N}=202)\end{array}$ & $\begin{array}{c}\text { Univariate OR } \\
(95 \% \mathrm{CI})\end{array}$ & p-value & $\begin{array}{l}\text { Multivariate OR } \\
\quad(95 \% \mathrm{CI})\end{array}$ & p-value \\
\hline Sex, male, $\%$ & 62.7 & 56.9 & 65.8 & $0.68(0.42-1.10)$ & 0.120 & $0.89(0.38-2.09)$ & 0.889 \\
\hline Age, mean (SD) & $15.95(1.41)$ & $15.7(1.5)$ & $16.1(1.4)$ & $0.85(0.69-1.04)$ & 0.116 & $0.78(0.54-1.13)$ & 0.780 \\
\hline \multicolumn{8}{|l|}{ Area of residence, $\%$} \\
\hline Large city & 11.3 & 51.4 & 42.1 & Ref & & Ref & \\
\hline Small city & 43.4 & 37.6 & 46.5 & $0.66(0.40-1.09)$ & 0.105 & $0.67(0.30-1.63)$ & 0.633 \\
\hline Rural & 45.3 & 11.0 & 11.4 & $0.79(0.37-1.72)$ & 0.555 & $0.95(0.27-3.38)$ & 0.933 \\
\hline \multicolumn{8}{|l|}{ School type, \% } \\
\hline Middle school & 35.7 & 40.4 & 33.2 & Ref & & Ref & \\
\hline Academic high school & 50.5 & 50.5 & 50.5 & $1.47(0.59-3.66)$ & 0.412 & $1.19(0.38-3.78)$ & 0.763 \\
\hline Vocational high school & 13.8 & 9.2 & 16.3 & $0.35(0.09-1.30)$ & 0.116 & $0.44(0.08-2.42)$ & 0.345 \\
\hline \multicolumn{8}{|l|}{ Residential type, \% } \\
\hline Living with family & 58.8 & 53.2 & 61.9 & Ref & & Ref & \\
\hline Living with relatives & 10.3 & 9.2 & 10.9 & $0.98(0.44-2.20)$ & 0.960 & $1.79(0.37-8.55)$ & 0.468 \\
\hline $\begin{array}{l}\text { Living with friends/alone/ } \\
\text { in a dormitory }\end{array}$ & 11.6 & 12.8 & 10.9 & $1.37(0.66-2.87)$ & 0.402 & $3.01(0.69-13.18)$ & 0.144 \\
\hline Living in a facility & 19.3 & 24.8 & 16.3 & $1.76(0.97-3.20)$ & 0.062 & $1.78(0.43-7.43)$ & 0.426 \\
\hline \multicolumn{8}{|l|}{ Socio-economic status, $\%$} \\
\hline Low & 25.4 & 33.0 & 21.3 & $1.48(0.77-2.85)$ & 0.239 & $0.37(0.09-1.47)$ & 0.158 \\
\hline Middle & 51.4 & 43.1 & 55.9 & $0.74(0.41-1.33)$ & 0.308 & $0.29(0.09-1.02)$ & 0.053 \\
\hline High & 23.2 & 23.9 & 22.8 & Ref & & Ref & \\
\hline \multicolumn{8}{|l|}{ Academic achievement, \% } \\
\hline Low & 30.5 & 41.3 & 24.8 & $2.38(1.32-4.30)$ & $0.004^{*}$ & $3.72(1.13-12.23)$ & $0.030^{*}$ \\
\hline Middle & 46.7 & 42.2 & 49.0 & $1.22(0.68-2.19)$ & 0.507 & $4.38(1.32-14.54)$ & $0.016^{*}$ \\
\hline High & 22.8 & 16.5 & 26.2 & Ref & & & \\
\hline Violence victimization & 47.9 & 60.6 & 41.1 & $2.20(1.37-3.54)$ & $0.001 *$ & $1.26(0.52-3.03)$ & 0.605 \\
\hline Perceived health & 63.0 & 50.5 & 69.8 & $0.44(0.27-0.71)$ & $0.001^{*}$ & $0.32(0.13-0.79)$ & $0.013^{*}$ \\
\hline Sleep satisfaction & 21.2 & 19.3 & 22.3 & $0.83(0.47-1.49)$ & 0.536 & $1.40(0.44-4.47)$ & 0.572 \\
\hline Problematic alcohol use & 43.4 & 51.4 & 39.1 & $1.65(1.03-2.63)$ & $0.038^{*}$ & $1.48(0.62-3.55)$ & 0.379 \\
\hline Poly-drug use & 71.4 & 80.7 & 66.3 & $2.13(1.22-3.72)$ & $0.008^{*}$ & $2.79(1.12-6.95)$ & $0.028^{*}$ \\
\hline Depressive mood & 61.4 & 89.9 & 46.0 & $10.44(5.28-20.65)$ & $<0.001^{*}$ & $19.79(5.15-75.96)$ & $<0.001^{*}$ \\
\hline
\end{tabular}

Multivariate odds ratios were calculated from multiple regression models including all variables in the Table. ${ }^{*} \mathrm{p}<0.05$. CI: confidence interval 
can be characterized as having mood disorders, stressful life events, interpersonal problems, poor social support, lonely lives, and feelings of hopelessness. Academic stress has received much attention in relation to youth suicidal behavior in South Korea, which is unique compared to research trends in other countries. ${ }^{16}$ It is reported that poor academic performance contributes to suicidal ideation and suicide attempts among Korean adolescents..$^{17,18}$

The limitations of this study include a lack of reliability and validity testing for the single-item surveys on depressive mood, perceived heath, and sleep satisfaction. Second, suicide attempts and drug use may have been underreported because of concerns over disclosing suicide attempts and illegal drug use. Finally, the frequency and risk level of drug use were not assessed in this study.

Even within the context of these limitations, the present study has clinical implications for the management of adolescent drug users. It would appear wise for those treating substance abuse to screen regularly for suicide attempts, ideation, and plans. In conducting such screening, assessments should include psychosocial factors such as hopelessness, poly-drug use, perceived health status, and academic-related stress. Preventive work should be directed toward the active treatment of drug use, depression, and physical health and reinforcing proper coping strategies for academic and other stress.

\section{Acknowledgments}

This work was supported by a clinical research grant (code 2015-04) from the Seoul National Hospital, Republic of Korea.

\section{REFERENCES}

1. WHO. Suicide Prevention (SUPRE) Information. Geneva: World Health Organization; 2012.

2. Park S, Cho SC, Kim BN, Kim JW, Yoo HJ, Hong JP. Increased use of lethal methods and annual increase of suicide rates in Korean adolescents: comparison with adolescents in the United States. J Child Psychol Psychiatr 2014;55:258-263.

3. OECD. OECD Health Data. Paris: OECD; 2014.

4. Hallfors DD, Waller MW, Ford CA, Halpern CT, Brodish PH, Iritani B.
Adolescent depression and suicide risk: association with sex and drug behavior. Am J Prev Med 2004;27:224-231.

5. Wong SS, Zhou B, Goebert D, Hishinuma ES. The risk of adolescent suicide across patterns of drug use: a nationally representative study of high school students in the United States from 1999 to 2009. Soc Psychiatry Psychiatr Epidemiol 2013;48:1611-1620.

6. Beautrais AL, Collings SCD, Ehrhardt P, Henare K. Suicide prevention: a review of evidence of risk and protective factors, and points of effective intervention. Wellington, New Zealand: Ministry of Health; 2005.

7. Suicide Prevention Resource Center, Rodgers P. Understanding Risk and Protective Factors for Suicide: A Primer for Preventing Suicide. Newton, MA: Education Development Center, Inc; 2011.

8. Cho BI. Drug Control Policy in Korea. Vancouver, BC: International Centre for Criminal Law Reform and Criminal Justice Policy; 2004.

9. Korea Centers for Disease Control and Prevention. Korea Youth Risk Behavior Web-Based Survey: Survey Summaries. Cheongwon: Korea Centers for Disease Control and Prevention; 2014.

10. Darke S, Ross J, Lynskey M, Teesson M. Attempted suicide among entrants to three treatment modalities for heroin dependence in the Australian Treatment Outcome Study (ATOS): prevalence and risk factors. Drug Alcohol Depend 2004;73:1-10.

11. Darke S, Williamson A, Ross J, Teesson M. Attempted suicide among heroin users: 12-month outcomes from the Australian Treatment Outcome Study (ATOS). Drug Alcohol Depend 2005;78:177-186.

12. Divin AL, Zullig KJ. The association between non-medical prescription drug use and suicidal behavior among United States adolescents. AIMS Public Health 2014;1:226-240.

13. Darke S, Ross J, Marel C, Mills KL, Slade T, Burns L, et al. Patterns and correlates of attempted suicide amongst heroin users: 11-year followup of the Australian treatment outcome study cohort. Psychiatry Res 2015;227:166-170.

14. Borges G, Walters EE, Kessler RC. Associations of substance use, abuse, and dependence with subsequent suicidal behavior. Am J Epidemiol 2000;151:781-789.

15. Pompili M, Serafini G, Innamorati M, Biondi M, Siracusano A, Di Giannantonio $\mathrm{M}$, et al. Substance abuse and suicide risk among adolescents. Eur Arch Psychiatry Clin Neurosci inpress.

16. Lee SY, Hog JS, Espelage DL. An ecological understanding of youth suicide in South Korea. School Psychol Intl 2010;31:531-546.

17. Cho SJ, Jeon HJ, Kim JK, Suh TW, Kim SU, Hahm BJ. Prevalence of suicide behaviors (suicidal ideation and suicide attempt) and risk factors of suicide attempts in junior and high school adolescents. J Korean Neuropsychiatr Assoc 2002;41:1142-1155.

18. Moon KS. The effect of academic stress on suicidal impulse in adolescence: mediating roles of parent and peer attachment. J Korean Assoc Child Stud 2006;27:143-158. 